\title{
NADES-based fractionation of biomass to produce raw material for the preparation of cellulose acetates
}

\author{
L. A. Soto-Salcido • I. Anugwom • L. Ballinas-Casarrubias • M. Mänttäri • \\ M. Kallioinen
}

Received: 8 January 2020/Accepted: 20 May 2020/Published online: 29 May 2020

(C) The Author(s) 2020

\begin{abstract}
Waste biomass (agave bagasse) and native birch wood were used as raw materials for a novel fractionation and derivation process to produce cellulose acetates (CAs). During the first stage of the fractionation process, a significant amount of hemicelluloses and lignin were dissolved from the biomass using a natural deep eutectic solvent (NADES) that consisted of a mixture of choline chloride and lactic acid with the molar ratio of 1:9. Then, the residual solid material was delignified by bleaching it with a mixture of acetic acid and sodium chlorite. The fractionation process generated differently purified pulps (celluloses) which were converted to CAs. The crystallinity index, polymerization degree, chemical
\end{abstract}

Electronic supplementary material The online version of this article (https://doi.org/10.1007/s10570-020-03251-1) contains supplementary material, which is available to authorized users.

L. A. Soto-Salcido $(\square)$

LUT SAWE Research Platform, LUT University,

Yliopistonkatu 34, 53850 Lappeenranta, Finland

e-mail: luis.soto.salcido@lut.fi

I. Anugwom

LUT RE-SOURCE Research Platform, LUT University,

Yliopistonkatu 34, 53850 Lappeenranta, Finland

e-mail: ikenna.anugwom@lut.fi

L. Ballinas-Casarrubias

Facultad de Ciencias Químicas, Universidad Autónoma

de Chihuahua, Chihuahua, Mexico

e-mail: mballinas@uach.mx composition, and thermal properties of the differently purified pulps and CAs were analyzed to evaluate the efficacy of the acetylation process and to characterize the CAs. The chemical derivation of the differently purified cellulose samples generated CAs with different degrees of substitution (DSs). The more purified the cellulose sample was, the higher its DS was. Moreover, some differences were observed between the acetylation efficiencies of birch and agave bagasse. Typically, cellulose purified from birch by treating it with NADES followed by bleaching was acetylated more completely ( $\mathrm{DS}=2.94)$ than that derived from agave bagasse ( $\mathrm{DS}=2.45$ ). These results revealed that using green solvents, such as NADES, to treat both agave bagasse (waste biomass) and birch wood, allowed pure fractions to be obtained from biomass, and thus, biomass could be valorized into products

\author{
M. Mänttäri · M. Kallioinen \\ LUT Department of Separation Science, LUT University, \\ Yliopistonkatu 34, 53850 Lappeenranta, Finland \\ e-mail: mika.manttari@lut.fi \\ M. Kallioinen \\ e-mail: mari.kallioinen@lut.fi
}


such as CAs, which present a wide range of applications.

Keywords Agave bagasse - Birch · Waste biomass valorization - Delignification - Green solvents . NADES

\section{Introduction}

Lignocellulosic biomasses have emerged as promising feedstock for the development of value-added products, and their abundancy, renewability, and biodegradability are some of their most attractive characteristics. Wheat straw, corn stover, sugarcane bagasse, cornhusk, cotton, oil palm empty fruit bunch, agave bagasse, rice husk, cereal straw, and different types of wood mainly comprise cellulose, hemicelluloses, and lignin, and have been used as raw materials for the production of chemicals, energy, and various materials.

Cellulose is the most abundant natural polymer on earth and the predominant component of lignocellulose (Wan Daud and Djuned 2015; Chen et al. 2016; Ruiz-Cuilty et al. 2018; Candido et al. 2017). Cellulose has been typically described as a linear chain of $\beta$ D-glucopyranosyl units linked by $1-4-\beta$-glucosidic bonds. Each anhydroglucose unit contains three reactive hydroxyl groups, which are susceptible to replacement by other chemical groups. Moreover, cellulose can be chemically modified to obtain materials with different physical and chemical properties for specific purposes.

Cellulose acetates (CAs), which are some of the most important derivatives of cellulose, are widely used for manufacturing textile fibers, cigarette filters, coats, films, composites, and membranes for filtration. CAs are synthesized via the acetylation of cellulose, where acetyl groups substitute the hydroxyl groups during esterification reactions where acetic acid, acetic anhydride, and sulfuric acid are typically used as the solvent, acetylating agent, and catalyst, respectively (Candido and Gonçalves 2016; Filho et al. 2005; El Nemr et al. 2015; Cerqueira et al. 2007; Das et al. 2014; Cao et al. 2018). CAs are typically produced from materials such as wood pulps or cotton, which contain highly pure cellulose, but are rather expensive (Cao et al 2018). Therefore, in the past decades, researchers focused their efforts toward the preparation of CAs from less expensive lignocellulosic biomasses, including corn fiber, rice hulls, and wheat straw (Biswas et al. 2006), rice husk (Das et al. 2014), agave bagasse (Soto-Salcido et al. 2018), sugarcane bagasse (Candido et al. 2017; Nakanishi et al. 2011), newspaper paper and mango seeds (Meireles et al. 2010), and Pinus sp. sawdust (Ballinas-Casarrubias et al. 2015).

The interactions of cellulose, hemicelluloses, and lignin result in the highly complex tridimensional structure of biomasses. Each of these compounds could be used for manufacturing value products. However, to be able to use them separately, biomasses need to be fractionated first (Li et al. 2018). Fractionation changes the micro- and macrostructure of biomasses and is typically performed by dissolving lignin and hemicellulose while leaving cellulose relatively unchanged and suitable for derivation (Chen et al. 2017). Kraft (sulphate) and sulfite cooking processes are the most commonly used pulping methods in the industry. Delignification occurs when lignin reacts with the sodium hydroxide in white liquor. The mass yields of these processes are considerably low, namely $50 \%$ or even lower, owing to the degradation and solubilization of carbohydrates during alkaline pulping at high temperature. The dominant pulping process, i.e., Kraft pulping, utilizes sodium sulfide under strongly alkaline conditions, and generates undesired sulfide derivatives and lignin, which contains sulfur (Idarraga et al. 1999; Santos et al. 2012; Robles et al. 2018; De La Torre et al. 2013). The Kraft cooking process is carried out in large cooking plants. When pure cellulose is the target compound, the pulp requires pre- and/or post-treatment. The large plants do not allow tailoring the pulp quality, and therefore, novel solvents for its fractionation have been developed. The fractionation processes studied and developed to date have aimed to minimize the loss and degradation of sugars, avoid the generation of harmful byproducts and the use of environmentally harmful reagents, and reduce the process cost (Chen et al. 2017). During the last decades, novel pretreatment methods using ionic liquids (IL) or organic solvents have been developed for the fractionation of biomasses. IL-based pretreatments have been demonstrated to be effective for the fractionation of several types of feedstock (PerezPimienta et al. 2016) however, their large-scale use is 
limited, mainly owing to the high cost of ILs and the need for complex and expensive recycling processes (Berglund et al. 2017).

Recently, a novel type of ionic solvents, known as deep eutectic solvents (DESs) have attracted the attention of researchers owing their high biodegradability and facile preparation process; moreover, DESs need not be purified after they are produced. Previous studies mostly defined DES as special mixtures of at least one hydrogen-bonding donor (HBD) and at least one hydrogen-bonding acceptor (HBA). The ability of DESs to donate and accept protons allows them to form hydrogen bonds with other compounds, and therefore, it improves their solvation properties. When natural compounds, such as amino acids, organic acids, sugars, urea, and choline derivatives, are used to synthesize DESs, the obtained solvents are commonly called natural deep eutectic solvents (NADESs). The natural origin of the components of NADESs prevails over some of the main drawbacks of common organic solvents, including their toxicity and high volatility (Santana et al. 2019; Faggian et al. 2016).

NADESs are prepared using heating, grinding, vacuum evaporation, and freeze-drying methods. When NADESs are prepared via heating, which is the simplest and most commonly used method, the components are mixed and heated until eutectic solvents form. The vacuum evaporation method requires that the components are first dissolved in water followed by their evaporation in vacuum. For the grinding method, the solid components are grinded in a mortar under nitrogen atmosphere until a transparent and homogeneous liquid is formed. When NADESs are prepared via freeze drying, the solid components are separately dissolved in water, and then, the solutions are mixed, frozen, and freeze-dried until a clear mixture is obtained (Van Osch et al. 2017).

The NADES that consists of choline chloride (CC) and lactic acid (LA) as the HBA and HBD, respectively, has been used to extract highly pure lignin from wood and is considered to be a promising solvent for the fractionation of biomasses and recovery of celluloses, because it requires milder cooking conditions than those used for conventional methods (Chen and Mu 2019; Mamilla et al. 2019; Satlewal et al. 2018; Li et al. 2018). According to the literature, different type of waste biomasses and pulps treated with NADESs present a wide range of delignification yields. For example, pretreatment using the NADES that consisted of a mixture of CC and LA with a molar ratio of approximately $1: 9$ in the temperature range of 60-90 ${ }^{\circ} \mathrm{C}$ was reported to decrease the lignin content of wheat straw to $14.6 \%$ (Jablonsky et al. 2015), potato peels and apple residues to $33 \%$ and $62 \%$, respectively (Procentese et al. 2018), hardwood Kraft pulp to $37.8 \%$ (Jablonsky et al. 2018), and corncob to $93.1 \%$ (Zhang et al. 2016). The waste biomass used in this study was agave bagasse, which is a solid waste generated during the production of tequila. Annually, approximately 350 kiloton of agave bagasse is produced in Mexico. This waste stream is typically discarded to landfills or is burnt. Furthermore, the usability of the agave bagasse waste biomass for the production of CA using the new NADES-based process was compared with that of silver birch (Betula pendula). The effect of the NADES pretreatment of agave bagasse on its delignification, crystallinity index (CrI), degree of polymerization (DP), hemicellulose removal, and cellulose accessibility for further chemical modification is currently unknown.

Therefore, the feasibility of the novel NADESbased fractionation process aimed at purifying cellulose, for further synthesis of CAs, from waste biomass, was studied. We hypothesized that the waste biomass could be valorized into CAs via NADES pretreatment followed by conventional acetylation. It was assumed that the NADES pretreatment of the waste biomass could be an efficient and mild fractionation process for producing cellulose-enriched pulp, which would be suitable for valorization into CAs.

\section{Materials and methods}

\section{Lignocellulosic biomass}

The agave bagasse was provided by Casa Cuervo (Jalisco, Mexico), a tequila manufacturer. The particle size of the bagasse was reduced using a hammer mill. The milled material was classified via sieving using 710 and $250 \mu \mathrm{m}$ meshes. The material that was collected using those meshes was selected for NADES pretreatment. The fibers were washed with acetone $(1: 10 \mathrm{w} / \mathrm{v})$ for $2 \mathrm{~h}$ at $50{ }^{\circ} \mathrm{C}$ to remove the dirt and extractives. The average size of the birch chips was $34 \times 16 \times 3 \mathrm{~mm}$, and they were not treated prior to 
the NADES pretreatment. Both biomasses were dried at $80{ }^{\circ} \mathrm{C}$ for $24 \mathrm{~h}$ before use.

\section{NADES preparation}

The NADES used in this study was prepared according to the procedure described by Esmaeili et al. (2018). A mixture of CC and LA with the molar ratio of 1:9 was vigorously stirred for $2 \mathrm{~h}$ at $70{ }^{\circ} \mathrm{C}$. The transparent and homogeneous mixture that was obtained was poured into a glass container, which was then sealed, where it was stored for further use.

\section{NADES pretreatment of biomasses}

First, $1000 \mathrm{~g}$ NADES was added to a pressureresistant glass recipient that contained $100 \mathrm{~g}$ biomass. The glass container was manually agitated to properly mix the reactants. Once the biomass was completely soaked with NADES, it was autoclaved for $5 \mathrm{~h}$ at $1.5 \mathrm{bar}$ and $121{ }^{\circ} \mathrm{C}$. Then, a $3 \mathrm{~L}$ aqueous ethanol solution (ethanol-to-water ratio of $2: 1 \mathrm{v} / \mathrm{v}$ ) was used to wash the pretreated biomass. Afterward, an abundant amount of water was used to wash and remove the impregnated NADES from the pulp. The pulp was dried for $24 \mathrm{~h}$ at $55^{\circ} \mathrm{C}$, and the yield of the pretreatment, delignification, and cellulose recovery were calculated as follows:

Yield $(\%)=\left(\frac{W_{2}}{W_{1}}\right) \times 100$,

where $W_{1}$ is the dry mass of the biomass $(\mathrm{g})$ and $W_{2}$ is the dry mass of the pulp $(\mathrm{g})$;

$\operatorname{Delignification}(\%)=\left[\frac{(L b-L p) \times \frac{Y}{100}}{L b}\right] \times 100$,

where $L b$ is the amount of lignin in the biomass $(\mathrm{g} / \mathrm{g})$, $L p$ is the amount of lignin in the pulp $(\mathrm{g} / \mathrm{g})$, and $Y$ is the mass pulp yield; and

Cellulose recovery $(\%)=\left[\frac{\left(\frac{C p \times Y}{100}\right)}{C b}\right] \times 100$,

where $C b$ is the cellulose content of the biomass $(\mathrm{g} / \mathrm{g})$ and $C p$ is the cellulose content of the pulp $(\mathrm{g} / \mathrm{g})$.
Acid-chlorite delignification

In an attempt to remove as much lignin as possible, the NADES-pretreated pulps were delignified using sodium chlorite and acetic acid. First, $20 \mathrm{~g}$ pulp and $640 \mathrm{~mL}$ deionized (DI) water were added to a $1 \mathrm{~L}$ Erlenmeyer flask, and the mixture was magnetically stirred; the temperature was set to $75{ }^{\circ} \mathrm{C}$. Then, $10 \mathrm{~g}$ sodium chlorite and $4 \mathrm{~mL}$ acetic acid were added to the flask and the mixture was agitated for $2 \mathrm{~h}$ while maintaining the same temperature. The produced delignified pulps were first washed with $160 \mathrm{~mL}$ acetone and then with DI water until their color changed from yellow to clear. Lastly, the pulps were dried at $55{ }^{\circ} \mathrm{C}$ for $24 \mathrm{~h}$.

\section{Determination of biomass and pulp composition}

The methods used to determine the cellulose, lignin, and hemicellulose composition of the biomass and pulps are described in Sect. 1.0 of the Supplementary Material (Anugwom et al. 2012; Sundberg et al. 1996; Sluiter et al. 2008; Willför et al. 2009).

Degree of polymerization

The average number of anhydroglucose units, known as the degree of polymerization (DP), determines the chain length of cellulose. The DP is an important factor that must be considered when designing cellulose derivatives owing to its effect on the mechanical, physical, chemical, and rheological properties of cellulose (Hilal et al. 2015). The effects of the NADES pretreatment and acid-chlorite delignification on the chain length were determined by measuring the DPs of the samples. We used the method described by Liu et al. (2016) who established a relationship between the intrinsic viscosity $([\eta])$ and molecular weight of cellulose dissolved in ILs. The pulps were added to a mixture of dimethyl sulfoxide and 1-ethyl-3methylimidazolium acetate $(1: 1 \mathrm{w} / \mathrm{w})$ and were allowed to dissolve at $80{ }^{\circ} \mathrm{C}$ for at least $24 \mathrm{~h}$. The concentration of the samples was $0.5 \%(0.005 \mathrm{~g} / \mathrm{mL})$. Then, the relative viscosities $\left(\eta_{r}\right)$ of the samples were determined using an SI analytics 532 20-II Ubbelohde viscometer at $40{ }^{\circ} \mathrm{C}$. The equations used to calculate $\eta_{r}$ and the specific viscosity $\left(\eta_{s p}\right)$ are included in Sect. 2.0 of the Supplementary Material (Shi et al. 2018). 
Acetylation reaction

The acetylation of the extracted cellulose was performed using the typical cellulose acetylation method described by Ballinas-Casarrubias et al. (2015). The method involved the use of acetic and sulfuric acids as solvent and catalyst, respectively in a temperaturecontrolled process. Briefly, first, cellulose was activated by adding acetic anhydride to it. Then, cellulosesulfate ester was formed upon the addition of sulfuric acid to the system. The reaction of acetic anhydride and sulfuric acid generated acetyl-sulfuric acid, which led to the formation of cellulose sulfate esters. During the acetylation reaction, a fraction of cellulose degraded owing to the hydrolysis of the glycosidic oxygen bonds in the acidic media. The distribution of the acetyl groups and degree of acetylation of cellulose were affected by the water content of the reaction mixture during hydrolysis (Ballinas-Casarrubias et al. 2015).

Four pulps with different lignin and hemicellulose contents were acetylated. First, $20 \mathrm{~g}$ pulp was collocated into a glass container; then, $55 \mathrm{~mL}$ acetic acid was added to it and the mixture was stirred manually every $10 \mathrm{~min}$ for $1 \mathrm{~h}$ at $38{ }^{\circ} \mathrm{C}$. A solution of $118.5 \mathrm{~mL}$ acetic acid and $0.12 \mathrm{~mL}$ sulfuric acid was subsequently added to the container, and the reactants were mixed for $1 \mathrm{~h}$ using a magnetic stirrer; the temperature of the reaction mixture was maintained at $38{ }^{\circ} \mathrm{C}$. Afterward, the mixture was cooled to $18{ }^{\circ} \mathrm{C}$, and a solution of $72 \mathrm{~mL}$ acetic anhydride and $0.24 \mathrm{~mL}$ sulfuric acid was added dropwise to it under constant stirring for $1 \mathrm{~h}$ at $18{ }^{\circ} \mathrm{C}$. Subsequently, the mixture was stirred for $1 \mathrm{~h}$ as its temperature was increased to $35{ }^{\circ} \mathrm{C}$. The viscosity of the mixture increased noticeably. Then, a solution of $72 \mathrm{~mL}$ acetic acid and $112 \mathrm{~mL}$ water was added to the container, and the mixture was stirred for $1 \mathrm{~h}$ at $38{ }^{\circ} \mathrm{C}$. The resulting acetylated lignocellulose material was filtrated and washed with abundant water until neutral $\mathrm{pH}$ was achieved. Lastly, the sample was dried at $55^{\circ} \mathrm{C}$ for $24 \mathrm{~h}$.

\section{Esterification degree}

The esterification degree (ED) was determined using the procedure reported by Candido and Gonçalves (2016). First, $5 \mathrm{~mL}$ of $0.25 \mathrm{M} \mathrm{NaOH}$ was added to an Erlenmeyer flask that contained $0.1 \mathrm{~g}$ acetylated lignocellulose sample. The mixture was allowed to react for $24 \mathrm{~h}$. Then, $10 \mathrm{~mL}$ of $0.25 \mathrm{M} \mathrm{HCl}$ was added to the flask and the content of the flask was mixed for 30 min. Afterward, the mixture was titrated using a $0.25 \mathrm{M} \mathrm{NaOH}$ solution and phenolphthalein as the indicator. The ED was calculated as follows:

$\% E D=\frac{\{[(V b i+V b t) \times \mu b-(V a \times \mu a)] \times M \times \mu a \times 100\}}{W}$

where $W$ is the mass of the sample $(\mathrm{g}), M$ is the molar weight of the acetyl group ( $43 \mathrm{~g} \mathrm{~mol}^{-1}$ ), $V b i$ is the volume of $\mathrm{NaOH}$ added to the system (L), Vbt is the volume of $\mathrm{NaOH}$ used for titration $(\mathrm{L}), \mu b$ is the concentration of $\mathrm{NaOH}(\mathrm{M}), V a$ is the volume of $\mathrm{HCl}$ added to the system (L), and $\mu a$ is the concentration of $\mathrm{HCl}(\mathrm{M})$.

\section{Characterization}

All samples were analyzed using X-ray powder diffraction (XRD), Fourier-transform infrared spectroscopy (FTIR) and thermogravimetric analysis (TGA). The analysis methods are described in Sect. 3.0 of the Supplementary Material.

\section{Results and discussion}

The following nomenclature was used for the pulps herein: Agave $\mathrm{N}$ and Birch $\mathrm{N}$ denote the NADESpretreated agave and birch pulps, respectively; Agave NB and Birch NB denote the NADES-pretreated and acid-chlorite-delignified agave and birch pulps, respectively; Agave NA and Birch NA denote the NADES-pretreated and acetylated pulps derived from agave bagasse and birch chips, respectively; and Agave NBA and Birch NBA denote the NADESpretreated, acid-chlorite-delignified, and acetylated pulps from agave bagasse and birch chips, respectively.

NADES pretreatment and acid-chlorite delignification

Different DESs and NADESs have been extensively used for feedstock fractionation processes. The efficiency of the pretreatment depends on several factors, including the type of biomass, HBA and HBD used, 
HBA/HBD ratio, DES/biomass ratio, and reaction conditions. Thus, the removal of hemicelluloses, delignification, and fractionation yield might vary depending on the experiment. High delignification yields have been reported when a mixture of $\mathrm{CC}$ and LA was used for the purification of cellulose (Jablonsky et al. 2015; Alvarez-Vasco et al. 2016). This might be attributed to the presence of chloride ions in CC. Smink et al. (2019) studied the effect of CC on the pulping of Eucalyptus chips and determined that the chloride ion was the active reactant for biomass conversion. Pretreatment using other feedstocks and different NADESs is discussed in Sect. 4.0 of the Supplementary Material (Zhao et al. 2018).

The cellulose, lignin, and hemicellulose content of the investigated biomasses and their respective pulps are summarized in Table 1. The initial compositions of the agave bagasse and native birch used in this study were consistent with the values reported in literature, which ranged from 42 to $47 \%, 21.3-27 \%$, and 19.3-20.5\% for cellulose, hemicelluloses, and lignin, respectively, for agave bagasse (Perez-Pimienta et al. 2013, 2015; Saucedo-Luna et al. 2010), and from 34.7 to $41 \%, 27.9-31.2 \%$, and $18.7-29.6 \%$ for cellulose, hemicelluloses, and lignin, respectively for native birch (Matsakas et al. 2018; Wen et al. 2013; Mirahmadi et al. 2010).

The NADES pretreatment and acid-chlorite delignification were aimed at increasing the production of cellulose-enriched pulps by degrading and dissolving the hemicelluloses and lignin. Jablonsky et al. (2018) efficiently removed lignin and hemicelluloses from birch after pretreatment using a LA and CC-based NADES, and achieved a delignification yield of $35 \%$, whereas the hemicellulose removal reached $82.5 \%$

Table 1 Content of main biopolymers in biomass samples. Here $\mathrm{N}$ and NB denote NADES-pretreated and NADESpretreated and acid-chlorite-delignified pulps, respectively, (see Table 2). Moreover, after acid-chlorite delignification, the decrease in the hemicellulose content was negligible, and, as expected, the lignin fraction decreased up to $77 \%$. This delignification yield was considerably higher than that reported by Wen et al. (2013) who used [C2mim][OAc], an IL, to pretreat birch. In addition, Wen et al. (2013) reported that the lignin and hemicellulose contents of the analyzed samples only decreased from 25.4 to $20.5 \%$ and from 29.9 to $12.8 \%$, respectively.

The NADES pretreatment resulted in the efficient delignification of agave bagasse, which led to the removal of up to $23.4 \%$ of the total lignin in the biomass. In addition, hemicelluloses were dissolved, which promoted the cellulose accessibility and its suitability for chemical modification. Compared with the pretreatment reported by Perez-Pimienta et al. (2016), who used the [C2mim][OAc] IL, under similar reaction conditions $\left(120^{\circ} \mathrm{C}\right.$ and $3 \mathrm{~h}$ of reaction time), lignin removal reached up to $16 \%$ and hemicellulose removal was almost the same. In addition to the improved delignification yield and hemicellulose removal, the NADES pretreatment of agave bagasse generated pulp that was readily available for chemical modification, with and without acid-chlorite delignification after pretreatment. Because NADESs are less toxic and more affordable than ILs, they are considered better alternatives for the pretreatment of biomasses.

In this study, the delignification yield of native birch after NADES pretreatment was higher than that of agave bagasse. The effectiveness of any pretreatment in the terms of delignification yield, hemicellulose removal, and cellulose recovery depends on the structure and composition of the biomass that is

where NADES is a natural deep eutectic solvent that consists of a mixture of choline chloride and lactic acid with the molar ratio of $1: 9$

\begin{tabular}{llrr}
\hline Sample & Cellulose $(\% \pm \mathrm{SD})$ & Lignin $(\% \pm \mathrm{SD})$ & Hemicelluloses $(\% \pm \mathrm{SD})$ \\
\hline Agave bagasse & $42.0 \pm 3.46$ & $18.8 \pm 0.53$ & $22.5 \pm 1.70$ \\
Agave N & $73.8 \pm 2.30$ & $14.4 \pm 0.55$ & $6.5 \pm 0.56$ \\
Agave NB & $88.5 \pm 1.27$ & $6.4 \pm 1.69$ & $7.2 \pm 0.15$ \\
Native birch & $38.4 \pm 4.51$ & $26.5 \pm 1.51$ & $30.9 \pm 5.22$ \\
Birch N & $63.6 \pm 1.60$ & $17.2 \pm 1.27$ & $5.4 \pm 0.69$ \\
Birch NB & $89.3 \pm 2.18$ & $3.9 \pm 0.56$ & $5.1 \pm 0.60$ \\
\hline
\end{tabular}


Table 2 Mass yield, delignification, hemicellulose removal, and cellulose recovery of natural deep eutectic solvent (NADES)pretreated and acid-chlorite delignified agave bagasse and birch chips

\begin{tabular}{|c|c|c|c|c|c|c|c|c|}
\hline \multirow[t]{2}{*}{ Biomass } & \multicolumn{4}{|c|}{ NADES pretreatment } & \multicolumn{4}{|c|}{ Acid-chlorite delignification } \\
\hline & $\begin{array}{l}\text { Mass } \\
\text { yield } \\
(\%)\end{array}$ & $\begin{array}{l}\text { Delignification } \\
(\%)\end{array}$ & $\begin{array}{l}\text { Hemicellulose } \\
\text { removal }(\%)\end{array}$ & $\begin{array}{l}\text { Cellulose } \\
\text { recovery } \\
(\%)\end{array}$ & $\begin{array}{l}\text { Mass } \\
\text { yield } \\
(\%)\end{array}$ & $\begin{array}{l}\text { Delignification } \\
(\%)\end{array}$ & $\begin{array}{l}\text { Hemicellulose } \\
\text { removal }(\%)\end{array}$ & $\begin{array}{l}\text { Cellulose } \\
\text { recovery } \\
(\%)\end{array}$ \\
\hline $\begin{array}{l}\text { Agave } \\
\text { bagasse }\end{array}$ & 59.5 & 23.4 & 71.1 & 62 & 75.4 & 55.5 & 0 & 50 \\
\hline $\begin{array}{l}\text { Birch } \\
\text { chips }\end{array}$ & 66.5 & 35 & 82.5 & 73 & 71.1 & 77.3 & 5.5 & 45 \\
\hline
\end{tabular}

subjected to it. The substructure of lignin comprises mainly syringyl (S) and guaiacyl $(\mathrm{G})$. The $S / G$ ratio has been demonstrated to be an important factor when defining biomass recalcitrance. The reactivity of the $\mathrm{G}$ aromatic ring is lower than that of the $\mathrm{S}$ one (Derkacheva, 2013), and thus, higher delignification yields after pretreatment could be obtained from biomasses that contain more $\mathrm{S}$ than $\mathrm{G}$ (Lupoi et al. 2015). Perez-Pimienta et al. (2016) reported that the $\mathrm{S} / \mathrm{G}$ ratio of untreated agave bagasse was 4.3 , which was considerably higher than that of untreated birch (2.4) (Derkacheva 2013). Thus, we hypothesized that another reason, such as the size of the fibers, could have led to the delignification yield of birch being higher than that of agave bagasse, although the S/G ratio of agave bagasse was higher than that of birch according to Derkacheva (2013).

Effect of NADES pretreatment and acid-chlorite delignification on DP

Numerous studies have reported the effects of the NADES pretreatments on the chain length of cellulose. The conditions of the pretreatment, such as the type of solvent, temperature, reaction time, and catalyst (if used), directly determine the DP of the resulting pulps. The DPs of the NADES-pretreated agave bagasse and birch were 479 and 755 , respectively. The DP of NADES-pretreated birch was higher than that of birch that underwent thermocatalytic treatment in the presence of $\mathrm{HCl}$, which was 450 (Laka and Chernyavskaya 2007). The milder effect of the NADES pretreatment on biomass delignification resulted in cellulose with longer chains. Hence, the accessibility to the hydroxyl groups that could be replaced by acetyl groups during esterification was increased. After acid-chlorite delignification, the DPs of agave bagasse and birch pulps increased from 479 to 550 and from 755 to 819 , respectively. Other researchers who used acid-chlorite to delignify Pinus radiata and cotton linters have also reported this effect and claimed that the increase in DP might have been caused by the formation of long chains between the fibers in the network via hydrogen bonds (Puri 1984; Kumar et al. 2013).

\section{Acetylation reaction}

The degree of substitution (DS) determines the average number of hydroxyl groups replaced by acetyl groups in the glucosidic units of cellulose. The DS values range from 0 to 3 , where 3 indicates the full substitution of unbranched CAs. The DS affects the solubility and also the mechanical, chemical, physical, and thermal properties of CAs. In addition, the biodegradability and crystallinity of CAs vary according to their DS (Filho et al. 2005; Ghareeb and Radke 2013). CAs with DS ranging from 2.2 to 2.7 consist mostly of cellulose diacetate (CDA). This cellulose derivative exhibits low toxicity and is soluble in a wide variety of common organic solvents, including acetone. The main drawback of CDA is its biodegradability. The DS of cellulose triacetate (CTA) ranges from 2.8 to 3.0 ; moreover, CTA presents low flammability, low toxicity, and better resistance to degradation by microorganisms than CDA, which are considered advantages (Cao et al. 2018; Wan Daud and Djuned 2015; Fan et al. 2013).

The hydrophobicity of the acetyl groups leads to their great solubility in organic solvents. CDA is soluble in acetone and tetrahydrofuran, and CTA is soluble in dichloromethane and chloroform. The 
solubility of CAs with low DS values in common organic solvents is low (De Freitas et al. 2017). The acetylation reaction efficiency depends on the accessibility of reactants to cellulose. Therefore, the replacement of the hydroxyl groups by acetyl groups starts in the amorphous region of cellulose owing to the crystalline structure of cellulose hindering chemical reactions (Wan Daud and Djuned 2015; Cheng et al. 2010). The DS depends on multiple factors, such as the type of pulp, catalyst, reaction time, and esterification method, and a summary of the DS values reported in the literature for different biomasses subjected to different acetylation methods is presented in section 5.0 of the Supplementary Material (Filho et al. 2007).

The DS of Agave NA and Agave NBA were 2.14 and 2.45 , respectively, and were both ascribed to the presence of CDA in these pulps. The DS values of Agave NA and Agave NBA were lower than those of Birch NA (2.74), and Birch NBA (2.94). The lower DS values of the agave bagasse-derived pulps was attributed to the lignin content of the agave bagasse pulps being higher than that of the birch-derived pulps. Lignin surrounds cellulose, and thus, it hinders the access of acetyl groups to the hydroxyl groups and diminishes the efficiency of the acetylation reaction (Biswas et al. 2006). Similarly, even though the DS of Birch NA was higher than those of Agave NA and Agave NBA, the number of substituted hydroxyl groups of Birch NA was lower than that of Birch NBA, which contained less lignin than Birch NA. The NADES-pretreated pulps obtained in this study were esterified before and after acid-chlorite pretreatment and their DS values are presented in Table 3.

The low DS values of CA do not translate into poor quality materials. CA with DS values ranging from 2.45 to 2.5 are preferred owing to their high solubility in many solvents (including acetone), and good melting properties, which render them useful for a variety of products (Candido et al. 2017). Thus, Agave NBA with the DS of 2.45 is preferred over birch with higher DS values for manufacturing more products, because the solubility of birch in commonly used solvents is limited. During the acetylation reaction, the amorphous lignin/hemicellulose matrix that surrounds the cellulose microfibrils, restricts the accessibility of reagents to cellulose. In addition, lignin and hemicelluloses contain hydroxyl groups that are susceptible to acetylation, and therefore, the chemical modification
Table 3 Cellulose acetates yield, esterification degrees (EDs), and degrees of substitution (DSs) of acetylated cellulignin pulps. Here NA and NBA denote NADES-pretreated and acetylated and NADES-pretreated, acid-chlorite-delignified, and acetylated pulps, respectively, where NADES is a natural deep eutectic solvent that consists of a mixture of choline chloride and lactic acid with the molar ratio of 1:9

\begin{tabular}{llll}
\hline Acetylated pulp & Yield $(\%)$ & ED $(\%)$ & DS \\
\hline Agave NA & 24.3 & 32.33 & 2.14 \\
Agave NBA & 33.7 & 37.13 & 2.45 \\
Birch NA & 33.5 & 41.50 & 2.74 \\
Birch NBA & 44.7 & 44.56 & 2.94 \\
\hline
\end{tabular}

of cellulose is hindered. The reaction rates of the lignocellulose components decreases as follows: lignin $>$ hemicelluloses $>$ cellulose (Hill et al. 1998).

It has been reported that the size of the agave bagasse fibers is important during cellulose valorization. Reagents reach cellulose via diffusion, and diffusion occurs more efficiently for powdered agave bagasse than for agave bagasse that consists of long fibers. The high lignin content of biomasses has been reported to inhibit the acetylation reaction or interfere with the esterification process, and therefore, would result in CAs with low DS values. For example, Meireles et al. (2010) attempted to acetylate newspaper paper with the lignin content of $24.65 \%$ and reported that the reaction was not effective until the newspaper paper was subjected to delignification (Cao et al. 2018; Tronc et al. 2007; Hill et al. 1998). NADES pretreatment improved the accessibility of cellulose, and thus, led to obtaining acetylated celluloses with higher DS values. Tronc et al. (2007) reported that powdered and long fibers of unpretreated acetylated agave bagasse presented the DS values of 0.34 and 1.17 , respectively. The DS of the agave bagassederived pulp in this study was as high as 2.14 , which was attributed to the presence of CDA in its structure, and that of Agave NB was 2.45.

\section{FTIR spectroscopy}

To assess the possible changes in the structure of biomasses during the synthesis of CAs, all samples were subjected to FTIR analysis. The fractionation of both biomasses and hydroxyl substitution by acetate groups during the acetylation reaction could be 
observed in the FTIR spectra of the samples. The spectra of agave bagasse and their respective pulps are depicted in Fig. 1, and those of native birch and its pulps are illustrated in Fig. 2. The biomasses (agave bagasse, and native birch) and their pulps (Agave N, Agave NB, Birch N, and Birch NB) presented similar behaviors.

The intensity of the broad peak at approximately $3350 \mathrm{~cm}^{-1}$ was assigned to the-stretching of the $-\mathrm{OH}$ group, which is characteristic of cellulose, and slightly decreased after the NADES pretreatment. This might be due to the removal of some of the hydroxyl groups of hemicellulose and lignin during NADES pretreatment. The intensity of the $1510 \mathrm{~cm}^{-1}$ peak, which is characteristic of the stretching or bending vibrations of the $\mathrm{C}=\mathrm{C}$ and $\mathrm{C}=\mathrm{O}$ bonds of lignin noticeably decreased in both the NADES-pretreated and NADES-pretreated and acid-chlorite delignified samples. The peaks at 1444 and $1431 \mathrm{~cm}^{-1}$ were assigned to the bending and scissoring of the $-\mathrm{CH}_{2}-$ groups. The intensity of the $1250 \mathrm{~cm}^{-1}$ peak, which is characteristic of the stretching of the $\mathrm{C}-\mathrm{O}$ bonds of lignin, decreased after NADES pretreatment. The intensity of the peak at approximately $1280-1300 \mathrm{~cm}^{-1}$, which is attributed to the bending of the $-\mathrm{CH}_{2}-$ groups of crystalline cellulose, increased significantly (Barud et al. 2008; Robles et al. 2018; Chen et al. 2016; De Freitas et al. 2017; Ruiz-Cuilty et al. 2018; Garside and Wyeth 2003; Candido and Gonçalves 2016).

The intensities of the broad peaks at $3350 \mathrm{~cm}^{-1}$ in the FTIR spectra of the acetylated pulps (Agave NA, Agave NBA, Birch NA, and Birch NBA) were significantly lower than those of the non-acetylated pulps, which indicated that the number of - $\mathrm{OH}$ groups decreased as they were substituted by acetyl groups

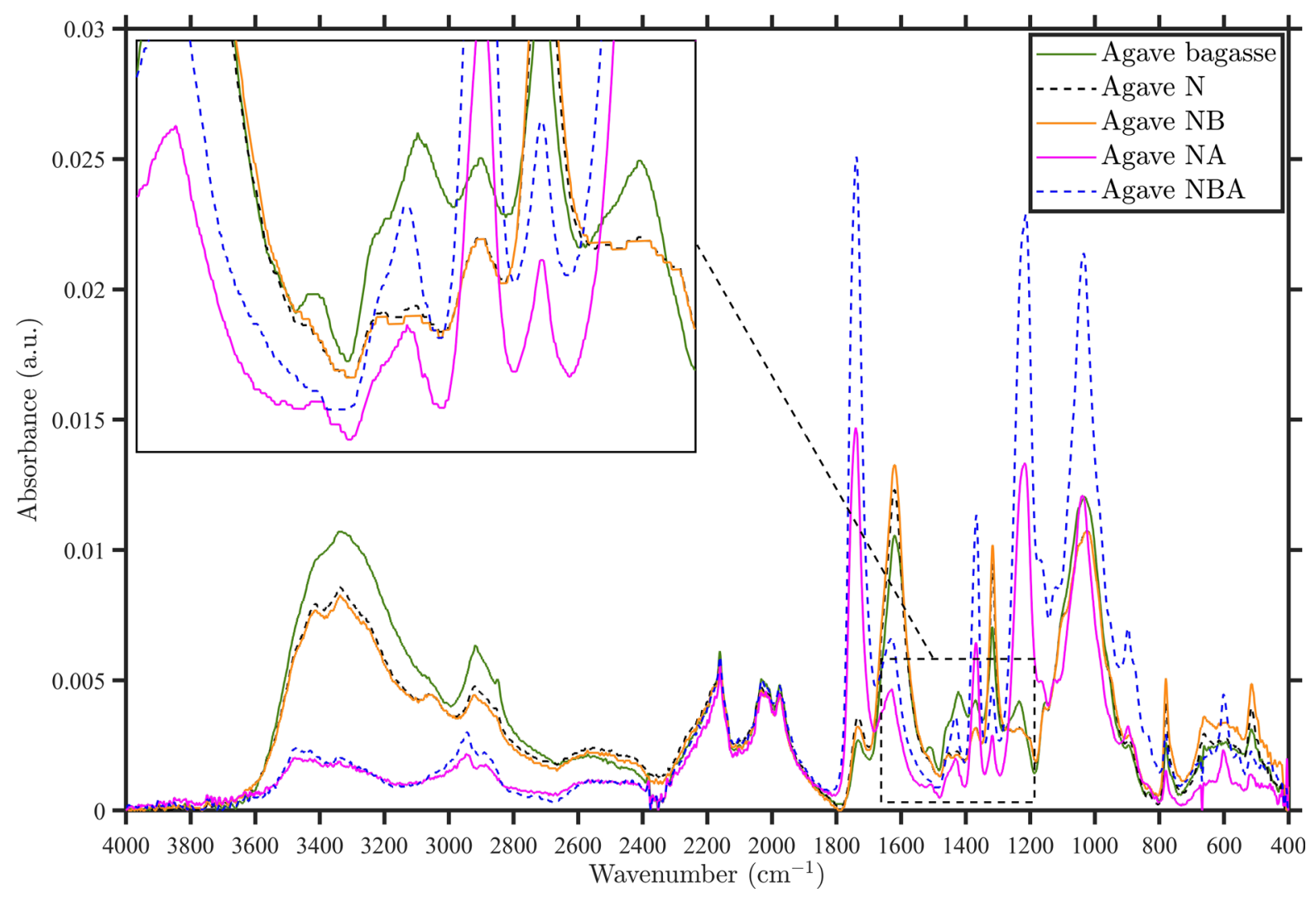

Fig. 1 Fourier-transform infrared spectra of Agave bagasse, Agave N, Agave NB, Agave NA, and Agave NBA; here Agave $\mathrm{N}$, Agave NB, Agave NA, and Agave NBA denote the NADESpretreated, NADES-pretreated and acid-chlorite-delignified, NADES-pretreated and acetylated, and NADES-pretreated, acid acid-chlorite-delignified, and acetylated pulps from agave bagasse, respectively, where NADES is a natural deep eutectic solvent that consists of a mixture of choline chloride and lactic acid with the molar ratio of $1: 9$ 


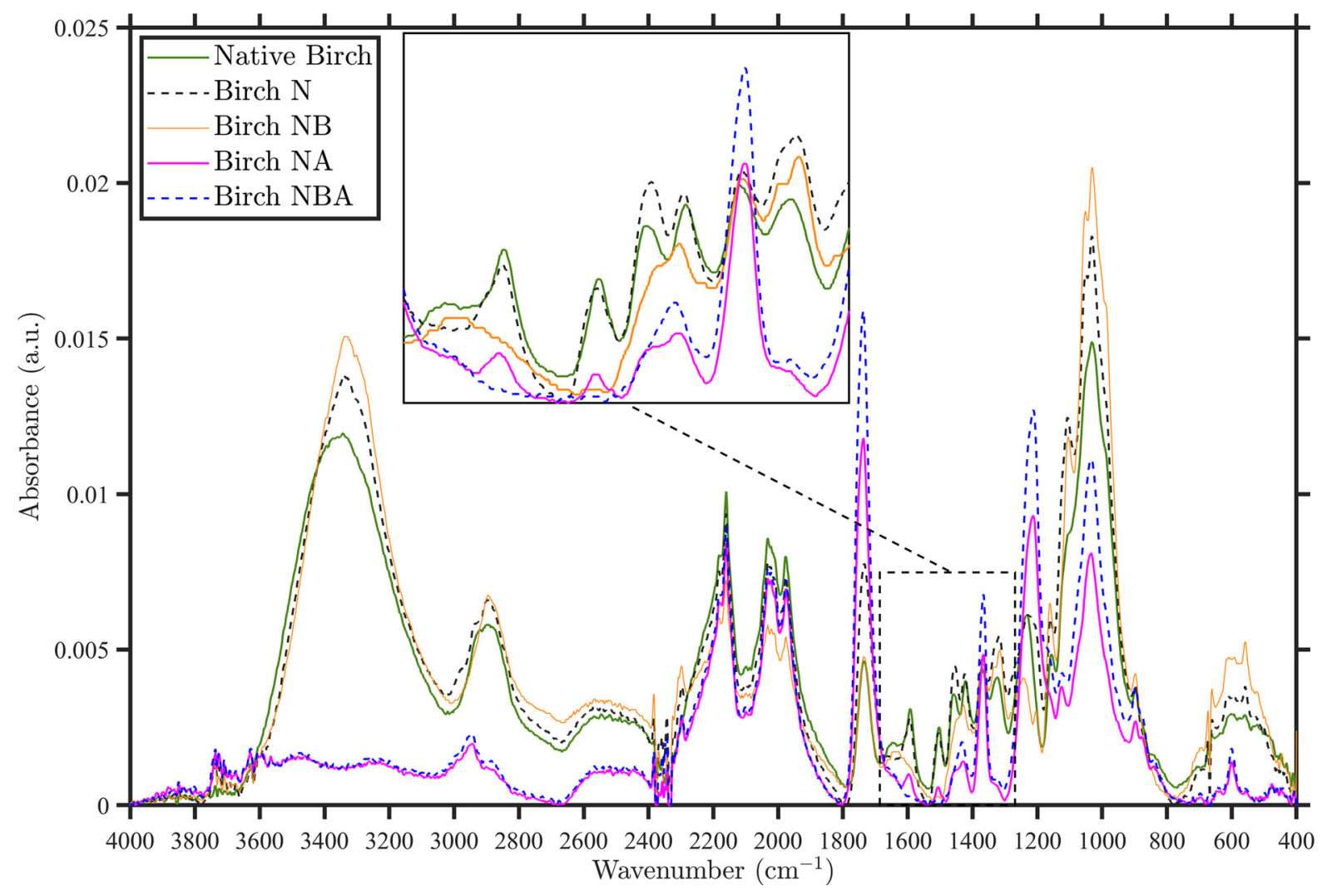

Fig. 2 Fourier-transform infrared spectra of Native Birch, Birch N, Birch NB, Birch NA, Birch NBA; here Birch N, Birch NB, Birch NA, and Birch NBA denote the NADES-pretreated, NADES-pretreated and acid-chlorite-delignified, NADES-pretreated and acetylated, and NADES-pretreated, acid acid-

during the esterification reaction. The intensities of the $1750 \mathrm{~cm}^{-1}$ peaks, which were assigned to the stretching of the $\mathrm{C}=\mathrm{O}$ ester carbonyl bonds present in the structure of the acetyl groups of the acetylated samples, were noticeably higher than those of the non-acetylated samples. The intensities of the $1360 \mathrm{~cm}^{-1}$ peaks, which were assigned to the $\mathrm{C}-\mathrm{H}$ bonds of the acetyl groups of the acetylated samples, were remarkably higher than those of the non-acetylated samples. The intensities of the $1240 \mathrm{~cm}^{-1}$ peaks in the spectra of the acetylated samples were higher than those of the non-acetylated samples owing to the stretching of the $\mathrm{C}-\mathrm{O}$ bonds of the acetyl groups linked to cellulose (Chen et al. 2016; De Freitas et al. 2017; Wan Daud and Djuned 2015; Luz et al. 2008; Candido et al. 2017; El Nemr et al. 2015). These results confirmed the removal of lignin and hemicelluloses from the pulps after NADES pretreatment and chlorite-delignified, and acetylated pulps from birch chips, respectively, where NADES is a natural deep eutectic solvent that consists of a mixture of choline chloride and lactic acid with the molar ratio of 1:9

acid-chlorite delignification. Moreover, the acetylation of the pulps was confirmed using the FTIR spectra, which revealed that the hydroxyl groups were replaced by acetyl groups.

Powder XRD analysis

The morphological changes and CrIs of the samples obtained when CAs were synthesized from biomasses were determined using XRD analysis. The $\mathrm{CrI}$ provides important information on the mechanical properties of materials (Zhao et al. 2016). Figure 3 presents the XRD pattern of native birch, its pulps, and acetylated pulps, and Fig. 4 illustrates the corresponding XRD patterns of the agave bagasse-based samples.

The patterns of Native Birch, Birch N, and Birch NB presented an intense peak at approximately $16^{\circ} 2 \theta$ corresponding to overlapped peaks of the 


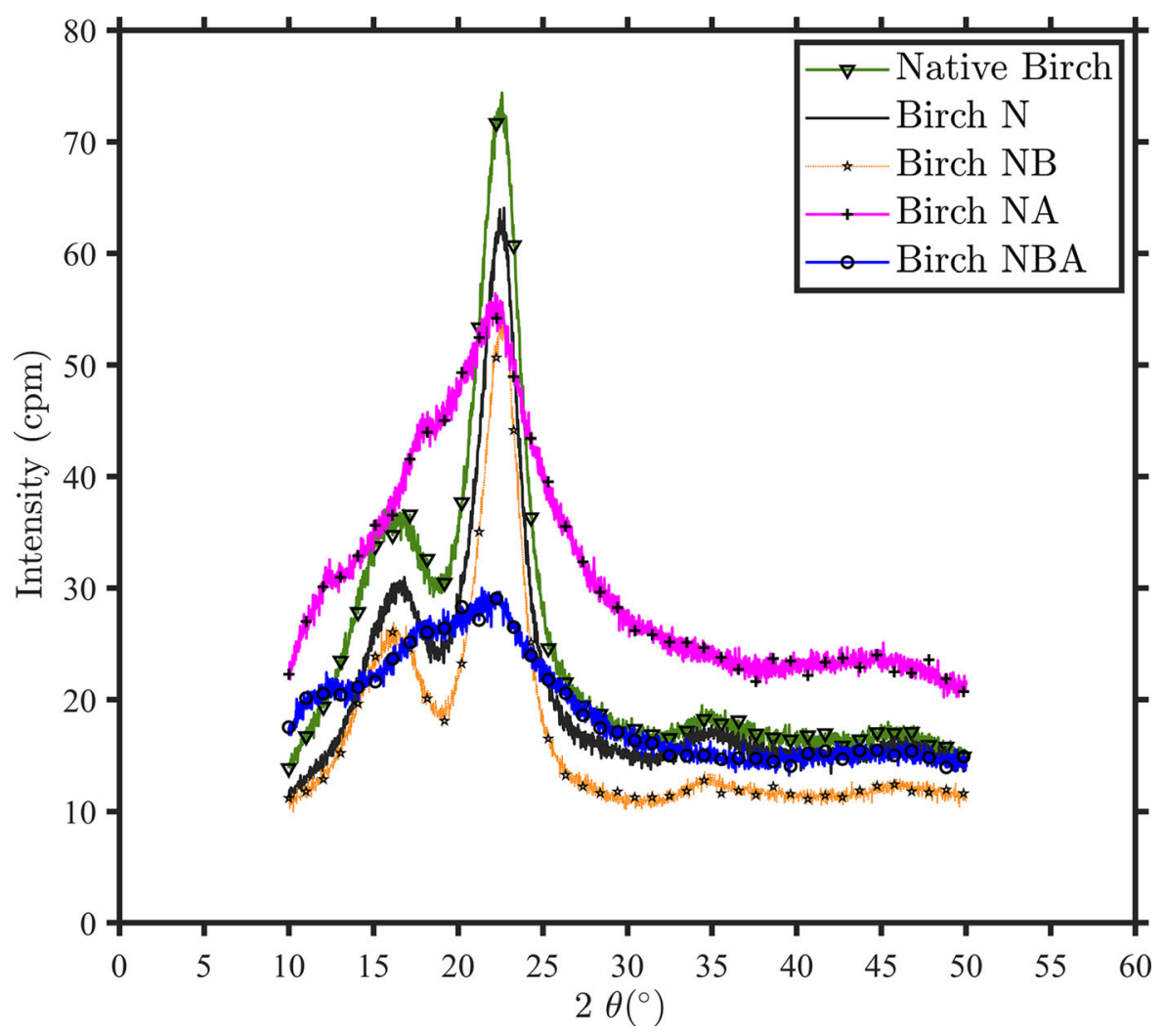

Fig. 3 X-ray diffraction patterns of Native birch, Birch N, Birch NB, Birch NA, and Birch NBA; here Birch N, Birch NB, Birch NA, and Birch NBA denote the NADES-pretreated, NADESpretreated and acid-chlorite-delignified, NADES-pretreated and acetylated, and NADES-pretreated, acid acid-chlorite-

crystallographic planes with Miller indices (1-10) and (110). The major intensity contributor to the peak at $23.0^{\circ} 2 \theta$, attributed to crystalline cellulose, corresponds to the (200) plane (French 2014; Pereira et al. 2020). In the $34.5^{\circ} 2 \theta$, is assigned to an overlapping of several peaks including (004) plane without this being the dominant contributor (French 2014). Whereas, the height of minimum intensity between the major peaks correspond to the amorphous material and it is located approximately at $18^{\circ} 2 \theta$ for cellulose I $\beta$ (French 2014; French and Santiago Cintrón 2013). The pretreated and delignified samples presented higher CrIs than native birch and agave bagasse owing to the removal of amorphous lignin and hemicelluloses from them during these processes. Ruiz-Cuilty et al. (2018) indicated that the crystallinity of Quercus sp. pulp samples was higher when their lignin content was lower. This was attributed to the degradation of amorphous cellulose during high-temperature delignified, and acetylated pulps from birch chips, respectively, where NADES is a natural deep eutectic solvent that consists of a mixture of choline chloride and lactic acid with the molar ratio of $1: 9$

$\left(180{ }^{\circ} \mathrm{C}\right)$ delignification. The CrIs of the samples were calculated using the method proposed by Segal et al. (1959), where the height intensity of the diffraction peak from the (200) plane, was divided by the height of minimum intensity of the amorphous fraction. The XRD patterns of the acetylated samples (Birch NA and Birch NBA) presented significant changes. According to Chen et al. (2016), the main diffraction peaks characteristic for semi-crystalline CAs are found at $8.3^{\circ}, 10.4^{\circ}$ and $13.3^{\circ} 2 \theta$, whereas the amorphous material is found at the diffraction peak $17.7^{\circ} 2 \theta$. The changes observed in the XRD pattern of the acetylated materials, are reported to have occurred during the acetylation of cellulose, this kind of disorder can be related to the substitution of acetyl group along the axes. This is often connected to an increased interfibrillar distance and microfibrillar structural breakage (Wan Daud and Djuned 2015). 


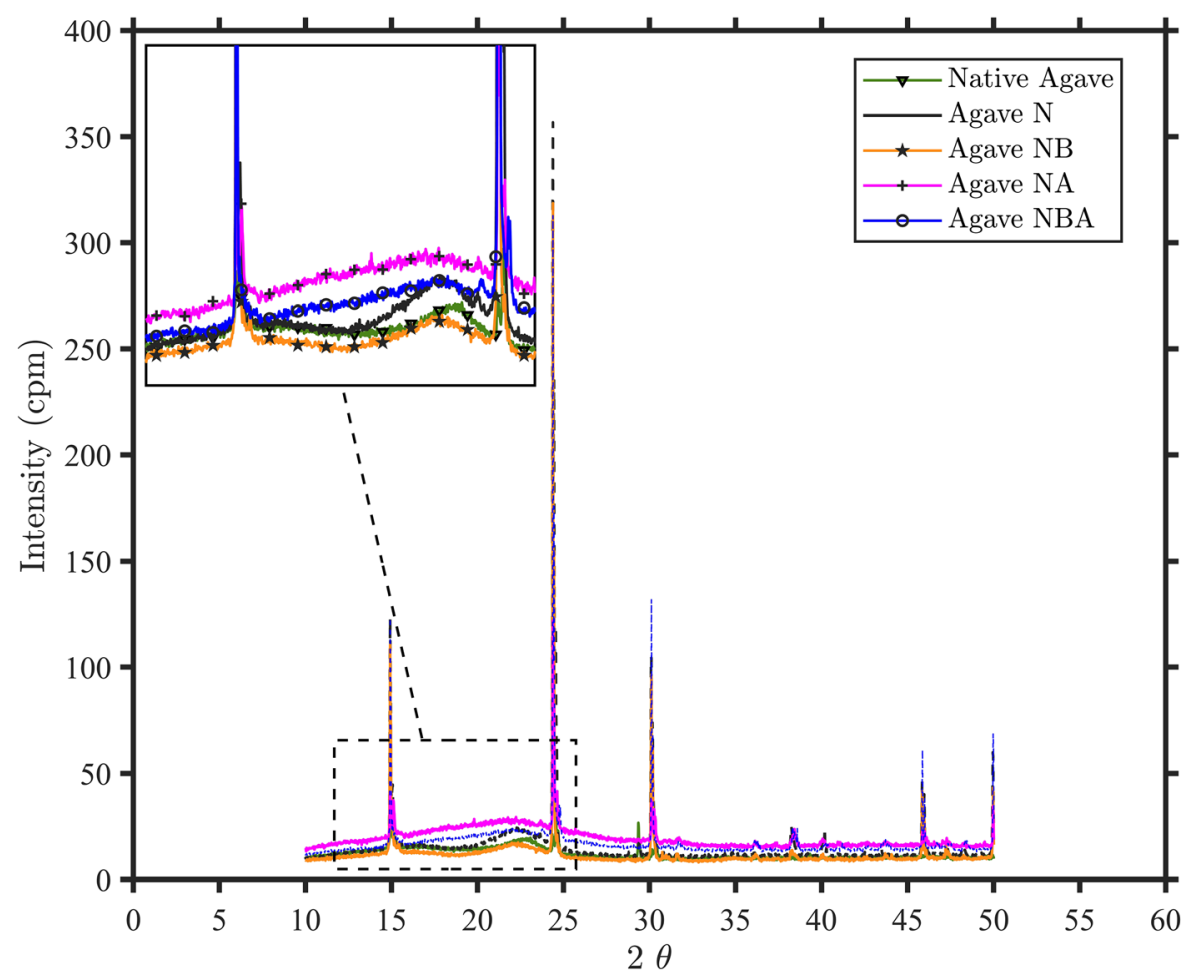

Fig. 4 X-ray diffraction patterns of Agave bagasse, Agave N, Agave NB, Agave NA, and Agave NBA; here Agave N, Agave NB, Agave NA, and Agave NBA denote the NADES-pretreated, NADES-pretreated and acid-chlorite-delignified, NADES-pretreated and acetylated, and NADES-pretreated, acid acid-

The XRD patterns of agave bagasse and its pulps included strong peaks at $2 \theta$ of $15^{\circ}, 24.5^{\circ}$, and $30.5^{\circ}$. These peaks were only visible in samples of agave bagasse collected after the production of tequila and were not observed in other varieties of agave (PerezPimienta et al. 2013). These peaks were ascribed to calcium oxalate monohydrate which commonly accumulates in the tissues of more than 215 plant families (Perez-Pimienta et al. 2015). The CrIs of all analyzed samples are summarized in Table 4 . The CrI of agave bagasse calculated in this study was $28 \%$, which was consistent with the CrI of $28.6 \%$ reported by PerezPimienta et al. (2013). The NADES pretreatment of agave bagasse resulted in high hemicellulose removal and partial delignification, which led to the increase in the CrIs of the NADES-pretreated samples. This was in agreement with the increase in the $\mathrm{CrI}$ of the agave bagasse pulp observed after organosolv pretreatment, where hemicellulose removal was considerable. chlorite-delignified, and acetylated pulps from agave bagasse, respectively, where NADES is a natural deep eutectic solvent that consists of a mixture of choline chloride and lactic acid with the molar ratio of $1: 9$

Table 4 Crystallinity indices of agave bagasse, birch chips, and their corresponding pulps; here $\mathrm{N}$, and NB, denote the NADES-pretreated, NADES-pretreated and acid-chloritedelignified, respectively, where NADES is a natural deep eutectic solvent that consists of a mixture of choline chloride and lactic acid with the molar ratio of 1:9

\begin{tabular}{ll}
\hline Sample & Crystallinity index $(\%)$ \\
\hline Native agave & 28 \\
Agave N & 36 \\
Agave NB & 35 \\
Native birch & 58 \\
Birch N & 61 \\
Birch NB & 66 \\
\hline
\end{tabular}

However, when $\left[\mathrm{C}_{2} \mathrm{C}_{1} \mathrm{Im}\right][\mathrm{OAc}]$, an IL, was used to pretreat agave bagasse, the CrIs of the samples decreased by approximately $42 \%$ and the amount of 
hemicelluloses in the samples after pretreatment was almost unchanged (Perez-Pimienta et al. 2017).

The CrIs of both biomasses increased after NADES pretreatment and acid-chlorite delignification. This was attributed to the removal of amorphous lignin and hemicelluloses, and the degradation of amorphous cellulose during the treatments (Ruiz-Cuilty et al. 2018). The higher the number of hydroxyl groups in the polymer chains, the more hydrogen bonds were formed, which improved the organization of chains and increased the amount of crystalline phases (De Freitas et al. 2017). The equation developed by Segal et al. (1959) cannot be used for acetylated materials, for this reason, CrIs of Agave NA, and NBA as well as Birch NA, and NBA samples are not presented in this work. However, De Freitas et al. (2017), reported that the CrIs of acetylated samples that presented higher DS values were lower than those of the samples that presented lower DS values.
TGA experiments

The TGA curves of native agave and its pulps are illustrated in Fig. 5, and those of native birch and its pulps are presented in Fig. 6. Three main thermal events, which are commonly observed for cellulose derivatives, were identified in the TGA curves of all samples. For Agave N and Agave NB, a slight weight loss was observed in the temperature range of $\mathrm{xx}-$ $180{ }^{\circ} \mathrm{C}$, which was attributed to the desorption of water and evaporation of volatile compounds (Candido et al. 2017; Das et al. 2014). Conversely, for Agave NA and Agave NBA this weight loss ended at approximately $140{ }^{\circ} \mathrm{C}$. This might be attributed to the content of volatile compounds present in the nonacetylated pulps being higher than that in acetylated pulps. The initial weight loss for native birch, Birch N and Birch NB occurred in the temperature range of $\mathrm{xx}-$ $230{ }^{\circ} \mathrm{C}$, and for Birch NA and Birch NBA in the temperature range of $\mathrm{xx}-150{ }^{\circ} \mathrm{C}$.

The second and major weight loss step occurred from $180{ }^{\circ} \mathrm{C}$ for agave bagasse, Agave $\mathrm{N}$ and Agave

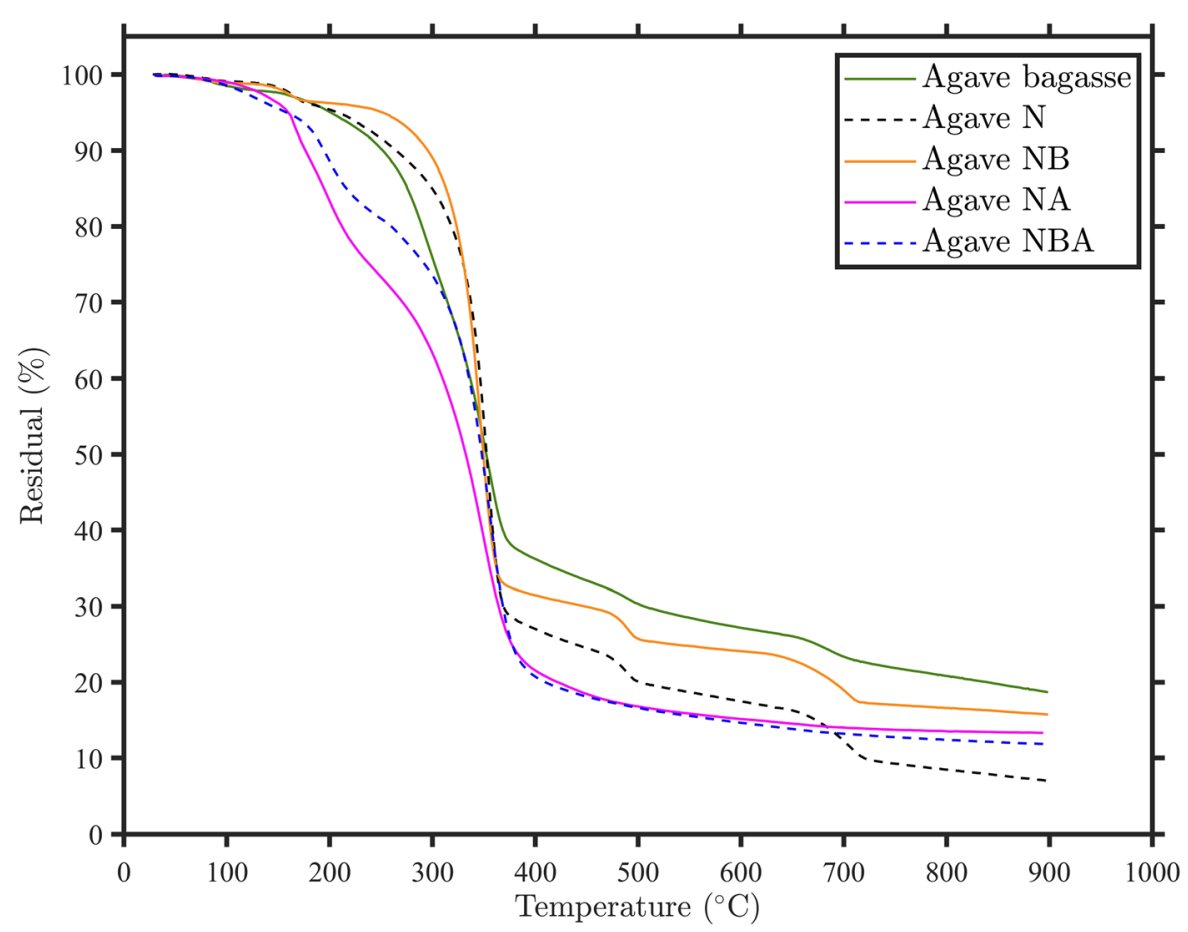

Fig. 5 Thermogravimetric analysis curves of Agave bagasse, Agave N, Agave NB, Agave NA, and Agave NBA, in the temperature range of $\mathrm{xx}-900{ }^{\circ} \mathrm{C}$; here Agave $\mathrm{N}$, Agave NB, Agave NA, and Agave NBA denote the NADES-pretreated, NADES-pretreated and acid-chlorite-delignified, NADES- pretreated and acetylated, and NADES-pretreated, acid acidchlorite-delignified, and acetylated pulps from agave bagasse, respectively, where NADES is a natural deep eutectic solvent that consists of a mixture of choline chloride and lactic acid with the molar ratio of $1: 9$ 


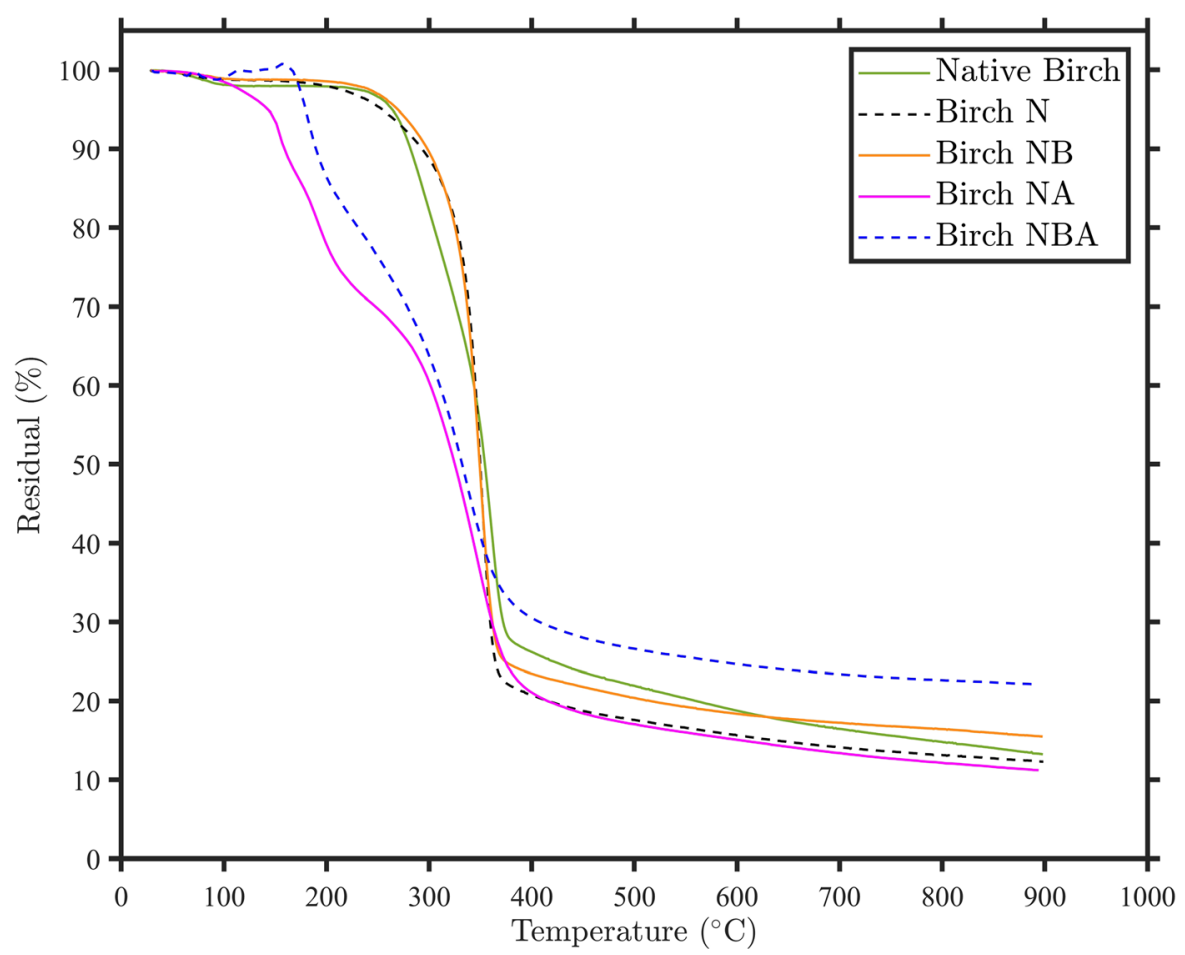

Fig. 6 Thermogravimetric analysis curves of Native birch, Birch N, Birch NB, Birch NA, and Birch NBA, in the temperature range of $\mathrm{xx}-900{ }^{\circ} \mathrm{C}$; here Birch $\mathrm{N}$, Birch $\mathrm{NB}$, Birch NA, and Birch NBA denote the NADES-pretreated, NADES-pretreated and acid-chlorite-delignified, NADES-

NB, $140{ }^{\circ} \mathrm{C}$ for Agave NA and Agave NBA, $230{ }^{\circ} \mathrm{C}$ for Native birch, Birch N, and Birch NB, and $150{ }^{\circ} \mathrm{C}$ for Birch NA, and Birch NBA, to approximately $380{ }^{\circ} \mathrm{C}$ for all samples. The pyrolytic decomposition of CAs occurred during this stage. Moreover, hemicelluloses decompose in the temperature range of approximately $180-230{ }^{\circ} \mathrm{C}$ owing to their amorphous structure (Meireles et al. 2010; Ruiz-Cuilty et al. 2018). Furthermore, lignin, which presents more complex structure, degrades in the temperature range of $190-900{ }^{\circ} \mathrm{C}$ (Candido et al. 2017) and the thermal degradation of cellulose occurs at approximately $350{ }^{\circ} \mathrm{C}$. This might explain the significant weight loss of the samples in the temperature range of $180-380{ }^{\circ} \mathrm{C}$ (Ruiz-Cuilty et al. 2018). As the temperature increased from 400 to $550{ }^{\circ} \mathrm{C}$, cellulose decomposed into $\mathrm{D}$-glucopyranose monomers, and then into free radicals (Das et al. 2014). At $200{ }^{\circ} \mathrm{C}$, the acetylated pulps presented a minor weight loss, which was attributed to the presence of a small quantity of xylan acetate in the samples owing to the acetylation of hemicelluloses pretreated and acetylated, and NADES-pretreated, acid acidchlorite-delignified, and acetylated pulps from birch chips, respectively; and NADES is a natural deep eutectic solvent that consisted of a mixture of choline chloride and lactic acid with the molar ratio of 1:9

(Meireles et al. 2010). Barud et al. (2008) assigned the peak at approximately $227{ }^{\circ} \mathrm{C}$ to the degradation of $\mathrm{CDA}$, and this peak is expected to be observed in the TGA curves of acetylated pulps with high DS. The third weight loss step occurred in the temperature range of $380-900{ }^{\circ} \mathrm{C}$, and was attributed to the carbonization of the degradation products (Candido et al. 2017; Fan et al. 2014). The high crystallinity of cellulose in the Agave N, Agave NB, Birch N, and Birch NB samples led to their thermal stability being higher than those of native birch, and agave bagasse, respectively. The Agave NBA, and Birch NBA samples were more thermally stable than Agave NA and Birch NA, and that could be attributed to the $\mathrm{CrI}$ and DS values of the NBA samples being higher than those of the NA samples (Chen et al. 2016; Barud et al. 2008). 


\section{Conclusions}

The goal of this study was to evaluate the feasibility of a novel fractionation process for the purification of cellulose for the production of CAs. Both waste biomass (agave bagasse) and native birch wood were used as raw materials for fractionation. Agave bagasse was selected because it is typically considered to be a waste and is only used for low value applications or discarded. The efficiencies of the fractionation process and acetylation reaction for both biomasses were compared. The fractionation process consisted of two steps: the pretreatment of biomass with a NADES and bleaching. The results confirmed our hypothesis that waste biomass could be valorized to CAs using a novel, mild, and simple NADES-based fractionation pretreatment prior to the acetylation of cellulose.

LA and CC-based NADES pretreatment was effective for dissolving hemicelluloses and lignin from the agave bagasse and birch samples. The hemicellulose removal and delignification were approximately $83 \%$ and $71 \%$ for the birch sample and $35 \%$ and $23 \%$ for the agave bagasse, respectively. The dissolution of hemicelluloses owing to the NADES pretreatment resulted in a cellulose-rich pulp that was readily available for chemical modification. Bleaching removed most of the residual lignin but did not significantly affect the hemicellulose content of the samples. The DS values of the acetylated pulps (celluloses) were higher when the purity of the cellulose in the pulp was higher. Moreover, the hydroxyl groups of the birch-derived cellulose were more completely acetylated than those of the agave bagasse-derived cellulose. The efficacy of the acetylation reaction was confirmed using FTIR analysis; the FTIR spectra of the samples that underwent acetylation revealed that the hydroxyl groups were substituted by acetyl groups. The acetylation of the agave bagasse-derived cellulose-enriched pulp lead to the formation of CDA, a desired material owing to its solubility and wide uses in the industry. Therefore, the proposed process could be used to valorize waste biomass (agave bagasse). In addition, our results suggested that, regardless of the source of the biomass, this novel process could purify cellulose that would be further used to prepare CAs.

Acknowledgments Open access funding provided by LUT University.
Open Access This article is licensed under a Creative Commons Attribution 4.0 International License, which permits use, sharing, adaptation, distribution and reproduction in any medium or format, as long as you give appropriate credit to the original author(s) and the source, provide a link to the Creative Commons licence, and indicate if changes were made. The images or other third party material in this article are included in the article's Creative Commons licence, unless indicated otherwise in a credit line to the material. If material is not included in the article's Creative Commons licence and your intended use is not permitted by statutory regulation or exceeds the permitted use, you will need to obtain permission directly from the copyright holder. To view a copy of this licence, visit http://creativecommons.org/licenses/by/4.0/.

\section{References}

Alvarez-Vasco C, Ma R, Quintero M, Guo M, Geleynse S, Ramasamy K, Wolcott M, Zhang X (2016) Unique lowmolecular-weight lignin with high purity extracted from wood by deep eutectic solvents (DES): a source of lignin for valorization. Green Chem 18:5133-5141. https://doi. org/10.1039/c6gc01007e

Anugwom I, Mäki-Arvela P, Virtanen P, Willför S, Sjöholm R, Mikkola J (2012) Selective extraction of hemicelluloses from spruce using switchable ionic liquids. Carbohydr Polym 87:2005-2011. https://doi.org/10.1016/j.carbpol. 2011.10.006

Ballinas-Casarrubias L, Saucedo-Acosta T, MacDonald-Pizaña K, Ruiz-Cuilty K, Nevárez-Moorillón G, Gutierréz-Méndez N, Torras-Font C, Chavez-Flores D, González-Sánchez G (2015) Organosolv pretreatment for cellulose recovery from sawdust for its ulterior use in membrane synthesis and operation. Desal Water Treat 56:3626-3639. https://doi. org/10.1080/19443994.2014.995720

Barud H, de Araújo JA, Santos D, de Assunção R, Meireles C, Cerqueira D, Rodrigues-Filho G, Ribeiro C, Messaddeq Y, Riberiro S (2008) Thermal behavior of cellulose acetate produced from homogeneous acetylation of bacterial cellulose. Thermochim Acta 471:61-69. https://doi.org/10. 1016/j.tca.2008.02.009

Berglund L, Anugwom I, Hedenström M, Aitomäki Y, Mikkola J, Oksman K (2017) Switchable ionic liquids enable efficient nanofibrillation of wood pulp. Cellulose 24:3265-3279. https://doi.org/10.1007/s10570-017-13542

Biswas A, Saha B, Lawton J, Shogren R, Willett J (2006) Process for obtaining cellulose acetate from agricultural byproducts. Carbohydr Polym 64:134-137. https://doi.org/ 10.1016/j.carbpol.2005.11.002

Cao L, Luo G, Tsang D, Chen H, Zhang S, Chen J (2018) A novel process for obtaining high quality cellulose acetate from green landscaping waste. J Clean Prod 176:338-347. https://doi.org/10.1016/j.jclepro.2017.12.077

Candido R, Godoy G, Gonçalves A (2017) Characterization and application of cellulose acetate synthesized from sugarcane bagasse. Carbohydr Polym 167:280-289. https://doi.org/ 10.1016/j.carbpol.2017.03.057 
Candido R, Gonçalves A (2016) Synthesis of cellulose acetate and carboxymethylcellulose from sugarcane straw. Carbohydr Polym 152:679-686. https://doi.org/10.1016/j. carbpol.2016.07.071

Cerqueira D, Filho G, Meireles C (2007) Optimization of sugarcane bagasse cellulose acetylation. Carbohydr Polym 69:579-582. https://doi.org/10.1016/j.carbpol.2007.01. 010

Chen J, Xu J, Wang K, Cao X, Sun R (2016) Cellulose acetate fibers prepared from different raw materials with rapid synthesis method. Carbohydr Polym 137:685-692. https:// doi.org/10.1016/j.carbpol.2015.11.034

Chen Y, Mu T (2019) Application of deep eutectic solvents in biomass pretreatment and conversion. Green Energy Environ. https://doi.org/10.1016/j.gee.2019.01.012

Chen H, Liu J, Chang X, Chen D, Xue Y, Liu P et al (2017) A review on the pretreatment of lignocellulose for high-value chemicals. Fuel Process Technol 160:196-206. https://doi. org/10.1016/j.fuproc.2016.12.007

Cheng H, Dowd M, Selling G, Biswas A (2010) Synthesis of cellulose acetate from cotton byproducts. Carbohydr Polym 80:449-452. https://doi.org/10.1016/j.carbpol. 2009.11.048

Das A, Ali A, Hazarika M (2014) Synthesis and characterization of cellulose acetate from rice husk: eco-friendly condition. Carbohydr Polym 112:342-349. https://doi.org/10.1016/j. carbpol.2014.06.006

Derkacheva O (2013) Estimation of aromatic structure contents in hardwood lignins from IR absorption spectra. J Appl Spectrosc 80:670-676. https://doi.org/10.1007/s10812013-9825-1

De Freitas R, Senna A, Botaro V (2017) Influence of degree of substitution on thermal dynamic mechanical and physicochemical properties of cellulose acetate. Ind Crop Prod 109:452-458. https://doi.org/10.1016/j.indcrop.2017.08. 062

De La Torre M, Hernández MA, M, Cabeza E, Tijero A, (2013) Organosolv lignin for biofuel. Ind Crop Prod 45:58-63. https://doi.org/10.1016/j.indcrop.2012.12.002

El Nemr A, Ragab S, El Sikaily A, Khaled A (2015) Synthesis of cellulose triacetate from cotton cellulose by using NIS as a catalyst under mild reaction conditions. Carbohydr Polym 130:41-48. https://doi.org/10.1016/j.carbpol.2015.04.065

Esmaeili M, Anugwom I, Mänttäri M, Kallioinen M (2018) Utilization of DES-lignin as a bio-based hydrophilicity promoter in the fabrication of antioxidant polyethersulfone membranes. Membranes 8:80. https://doi.org/10.3390/ membranes 8030080

Faggian M, Sut S, Perissutti B, Baldan V, Grabnar I, Dall'Acqua $S$, (2016) Natural deep eutectic solvents (NADES) as a tool for bioavailability improvement: pharmacokinetics of Rutin dissolved in proline/glycine after oral administration in rats: possible application in nutraceuticals. Molecules 21:1531. https://doi.org/10.3390/molecules21111531

Fan G, Liao C, Fang T, Luo S, Song G (2014) Amberlyst 15 as a new and reusable catalyst for the conversion of cellulose into cellulose acetate. Carbohydr Polym 112:203-209. https://doi.org/10.1016/j.carbpol.2014.05.082

Fan G, Wang M, Liao C, Fang T, Li J, Zhou R (2013) Isolation of cellulose from rice straw and its conversion into cellulose acetate catalyzed by phosphotungstic acid. Carbohydr
Polym 94:71-76. https://doi.org/10.1016/j.carbpol.2013. 01.073

Filho G, de Assunção R, Vieira J, Meireles C, Cerqueira D, da Silva BH et al (2007) Characterization of methylcellulose produced from sugar cane bagasse cellulose: crystallinity and thermal properties. Polym Degrad Stab 92:205-210. https://doi.org/10.1016/j.polymdegradstab.2006.11.008

Filho G, da Silva R, da Silva MC, de Assunção R, Otaguro H (2005) Water flux through blends from waste materials: cellulose acetate (from sugar cane bagasse) with polystyrene (from plastic cups). J Appl Polym Sci 96:516-522. https://doi.org/10.1002/app.21474

French A (2014) Idealized powder diffraction patterns for cellulose polymorphs. Cellulose. https://doi.org/10.1007/ s10570-013-0030-4

French A, Santiago Cintrón M (2013) Cellulose polymorphy, crystallite size, and the Segal Crystallinity Index. Cellulose. https://doi.org/10.1007/s10570-012-9833-y

Garside P, Wyeth P (2003) Identification of cellulosic fibres by FTIR spectroscopy-thread and single fibre analysis by attenuated total reflectance. Stud Conserv 48:269-275. https://doi.org/10.1179/sic.2003.48.4.269

Ghareeb H, Radke W (2013) Separation of cellulose acetates by degree of substitution. Polym 54:2632-2638. https://doi. org/10.1016/j.polymer.2013.03.041

Hilal N, Ismail A, Wright C (2015) Membrane fabrication. Florida, Boca Raton

Hill C, Jones D, Strickland G, Cetin N (1998) Kinetic and mechanistic aspects of the acetylation of woodwith acetic anhydride. Holzforschung 52:623-629. https://doi.org/10. 1515/hfsg.1998.52.6.623

Idarraga G, Ramos J, Zuñiga V, Sahin T, Young RA (1999) Pulp and paper from blue agave waste from tequila production. J Agric Food Chem 47(10):4450-4455

Jablonsky M, Škulcová A, Kamenská L, Vrška M, Šíma J (2015) Deep eutectic solvents: fractionation of wheat straw. Bioresources. https://doi.org/10.15376/biores.10.4.80398047

Jablonsky M, Majova V, Skulcova A, Haz A (2018) Delignification of pulp using deep eutectic solvents. J Hyg Eng Des 22:76-81

Kumar R, Hu F, Hubbell C, Ragauskas A, Wyman C (2013) Comparison of laboratory delignification methods, their selectivity, and impacts on physiochemical characteristics of cellulosic biomass. Bioresource Technol 130:372-381. https://doi.org/10.1016/j.biortech.2012.12.028

Laka M, Chernyavskaya S (2007) Obtaining microcrystalline cellulose from softwood and hardwood pulp. Bioresources 2:583-589. https://doi.org/10.15376/biores.2.4.583-589

Li A, Hou X, Lin K, Zhang X, Fu M (2018) Rice straw pretreatment using deep eutectic solvents with different constituents molar ratios: biomass fractionation, polysaccharides enzymatic digestion and solvent reuse. J Biosci Bioeng 126:346-354. https://doi.org/10.1016/j. jbiosc.2018.03.011

Liu J, Zhang J, Zhang B, Zhang X, Xu L, Zhang J et al (2016) Determination of intrinsic viscosity-molecular weight relationship for cellulose in BmimAc/DMSO solutions. Cellulose 23:2341-2348. https://doi.org/10.1007/s10570016-0967-1 
Lupoi J, Singh S, Parthasarathi R, Simmons B, Henry R (2015) Recent innovations in analytical methods for the qualitative and quantitative assessment of lignin. Renew Sust Energ Rev 49:871-906. https://doi.org/10.1016/j.rser. 2015.04.091

Luz S, Del Tio J, Rocha G, Gonçalves A, Del'Arco A, (2008) Cellulose and cellulignin from sugarcane bagasse reinforced polypropylene composites: effect of acetylation on mechanical and thermal properties. Compos Part A-Appl S 39:1362-1369. https://doi.org/10.1016/j.compositesa. 2008.04.014

Mamilla J, Novak U, Grilc M, Likozar B (2019) Natural deep eutectic solvents (DES) for fractionation of waste lignocellulosic biomass and its cascade conversion to valueadded bio-based chemicals. Biomass Bioenerg 120:417-425. https://doi.org/10.1016/j.biombioe.2018.12. 002

Matsakas L, Nitsos C, Raghavendran V, Yakimenko O, Persson $\mathrm{G}$, Olsson E et al (2018) A novel hybrid organosolv: steam explosion method for the efficient fractionation and pretreatment of birch biomass. Biotechnol Biofuels. https:// doi.org/10.1186/s13068-018-1163-3

Meireles C, Filho G, Fernandes Ferreira M Jr, Cerqueira D, Assunção R, Ribeiro E, et al (2010) Characterization of asymmetric membranes of cellulose acetate from biomass: newspaper and mango seed. Carbohydr Polym 80:954-961. https://doi.org/10.1016/j.carbpol.2010.01. 012

Mirahmadi K, Kabir M, Jeihanipour A, Karimi K, Taherzadeh M (2010) Alkaline pretreatment of spruce and birch to improve bioethanol and biogass production. BioResources. https://doi.org/10.1002/jctb.3695

Nakanishi S, Gonçalves A, Rocha G, Ballinas M, Gonzalez G (2011) Obtaining polymeric composite membranes from lignocellulosic components of sugarcane bagasse for use in wastewater treatment. Desal Water Treat 27:66-71. https:// doi.org/10.5004/dwt.2011.2053

Pereira P, Ornaghi Júnior H, Coutinho L, Duchemin B, Cioffi M (2020) Obtaining cellulose nanocrystals from pineapple crown fibers by free-chlorite hydrolysis with sulfuric acid: physical, chemical and structural characterization. Cellulose. https://doi.org/10.1007/s10570-020-03179-6

Perez-Pimienta J, Lopez-Ortega M, Varanasi P, Stavila V, Cheng G, Singh S, Simmons B (2013) Comparison of the impact of ionic liquid pretreatment on recalcitrance of agave bagasse and switchgrass. Bioresource Technol 127:18-24. https://doi.org/10.1016/j.biortech.2012.09.124

Perez-Pimienta J, Lopez-Ortega M, Chavez-Carvayar J, Varanasi P, Stavila V, Cheng G et al (2015) Characterization of agave bagasse as a function of ionic liquid pretreatment. Biomass Bioenerg 75:180-188. https://doi.org/10.1016/j. biombioe.2015.02.026

Perez-Pimienta J, Flores-Gómez C, Ruiz H, Sathitsuksanoh N, Balan V, da Costa SL et al (2016) Evaluation agave bagasse recalcitrance using AFEX, autohydrolysis, and ionic liquid pretreatments. Bioresource Technol 211:216-223. https://doi.org/10.1016/j.biortech.2016.03. 103

Perez-Pimienta J, Vargas-Tah A, López-Ortega K, MedinaLópez Y, Mendoza-Pérez J, Avila S et al (2017) Sequential enzymatic saccharification and fermentation of ionic liquid and organosolv pretreated agave bagasse for ethanol production. Bioresource Technol 225:191-198. https://doi. org/10.1016/j.biortech.2016.11.064

Procentese A, Raganati F, Olivieri G, Russo M, Rehmann L, Marzocchella A (2018) Deep eutectic solvents pretreatment of agro-industrial food waste. Biotechnol Biofuels. https://doi.org/10.1186/s13068-018-1034-y

Puri V (1984) Effect of crystallinity and degree of polymerization of cellulose on enzymatic saccharification. Biotechnol Bioeng 26:1219-1222. https://doi.org/10.1002/ bit. 260261010

Ruiz-Cuilty K, Ballinas-Casarrubias L, de San R, Miguel E, de Gyves J, Robles-Venzor J, González-Sánchez G (2018) Cellulose recovery from Quercus sp. sawdust using ethanosolv pretreatment. Biomass Bioenerg 111:114-124. https://doi.org/10.1016/j.biombioe.2018.02.004

Robles E, Fernández-Rodríguez J, Barbosa A, Gordobil O, Carreño N, Labidi J (2018) Production of cellulose nanoparticles from blue agave waste treated with environmentally friendly processes. Carbohydr Polym 183:294-302. https://doi.org/10.1016/j.carbpol.2018.01. 015

Santana A, Mora-Vargas J, Guimarães T, Amaral C, Oliveira A, Gonzalez M (2019) Sustainable synthesis of natural deep eutectic solvents (NADES) by different methods. J Mol Liq 293:111452. https://doi.org/10.1016/j.molliq.2019.111452

Santos R, Jameel H, Chang H, Hart P (2012) Kinetics of hardwood carbohydrate degradation during Kraft pulp cooking. Ind Eng Chem Res. https://doi.org/10.1021/ie301071n

Satlewal A, Agrawal R, Bhagia S, Sangoro J, Ragauskas A (2018) Natural deep eutectic solvents for lignocellulosic biomass pretreatment: recent developments, challenges and novel opportunities. Biotechnol Adv 36:2032-2050. https://doi.org/10.1016/j.biotechadv.2018.08.009

Saucedo-Luna J, Castro-Montoya A, Rico J, Campos-Garcia J (2010) Optimization of acid hydrolysis of bagasse from agave tequilana weber. Rev Mex Ing Quim 9:91-97

Segal L, Creely J, Martin A, Conrad C (1959) An empirical method for estimating the degree of crystallinity of native cellulose using the X-ray diffractometer. Text Res J 29:786-794. https://doi.org/10.1177/00405175590290 1003

Sluiter A, Hames B, Ruiz R, Scarlata C, Sluiter J, Templeton D, Crocker D (2008) Determination of structural carbohydrates and lignin in biomass. NREL laboratory analytical procedures for standard biomass analysis. Colorado, USA. https://www.nrel.gov/docs/gen/fy13/42618.pdf

Shi S, Zhang M, Ling C, Hou W, Yan Z (2018) Extraction and characterization of microcrystalline cellulose from waste cotton fabrics via hydrothermal method. Waste Manage 82:139-146. https://doi.org/10.1016/j.wasman.2018.10. 023

Smink D, Juan A, Schuur B, Kersten S (2019) Understanding the role of choline chloride in deep eutectic solvents used for biomass delignification. Ind Eng Chem Res 58:16348-16357. https://doi.org/10.1021/acs.iecr. $9 \mathrm{~b} 03588$

Soto-Salcido L, González-Sánchez G, Rocha-Gutierrez B, Peralta-Perez R, Zavala-Díaz F, Ballinas-Casarrubias L (2018) Preparation, characterization and performance of acetylated cellulignin membranes obtained by green methods 
from biomass. Desal 430:186-196. https://doi.org/10. 1016/j.desal.2017.12.017

Sundberg A, Sundberg K, Lillandt C, Holmbom B (1996) Determination of hemicelluloses and pectins in wood and pulp fibers by acid methanolysis and gas chromatography. Nord Pulp Pap Res J 4:216-226

Tronc E, Hernández-Escobar C, Ibarra-Gómez R, EstradaMonje A, Navarrete-Bolaños J, Zaragoza-Contreras E (2007) Blue agave fiber esterification for the reinforcement of thermoplastic composites. Carbhydr Polym 67:245-255. https://doi.org/10.1016/j.carbpol.2006.05.027

Van Osch D, Kollau L, van den Bruinhorst A, Asikainen S, Rocha M, Kroon M (2017) Ionic liquids and deep eutectic solvents for lignocellulosic biomass fractionation. Phys Chem Chem Phys 19:2636-2665. https://doi.org/10.1039/ c6cp07499e

Wan Daud W, Djuned F (2015) Cellulose acetate from oil palm empty fruit bunch via a one step heterogeneous acetylation. Carbohydr Polym 132:252-260. https://doi.org/10.1016/j. carbpol.2015.06.011

Wen J, Sun S, Xue B, Sun R (2013) Quantitative structures and thermal properties of birch lignins after ionic liquid pretreatment. J Agric Food Chem 61:635-645. https://doi.org/ $10.1021 /$ jf3051939
Willför S, Pranovich A, Tamminen T, Puls J, Laine C, Suurnäkki A et al (2009) Carbohydrate analysis of plant materials with uronic acid-containing polysaccharides-a comparison between different hydrolysis and subsequent chromatographic analytical techniques. Ind Crop Prod 29:571-580. https://doi.org/10.1016/j.indcrop.2008.11. 003

Zhao L, Jiang J, Lu J (2016) Effect of thermal expansion at low temperature on mechanical properties of Birch wood. Cold Reg Sci Technol 126:61-65. https://doi.org/10.1016/j. coldregions.2016.03.008

Zhao Z, Chen X, Ali M, Abdeltawab A, Yakout S, Yu G (2018) Pretreatment of wheat straw using basic ethanolaminebased deep eutectic solvents for improving enzymatic hydrolysis. Bioresour Technol 263:325-333. https://doi. org/10.1016/j.biortech.2018.05.016

Zhang C, Xia S, Ma P (2016) Facile pretreatment of lignocellulosic biomass using deep eutectic solvents. Bioresource Technol 219:1-5. https://doi.org/10.1016/j.biortech.2016. 07.026

Publisher's Note Springer Nature remains neutral with regard to jurisdictional claims in published maps and institutional affiliations. 